\title{
Linear Systems over Join-Blank Algebras
}

\author{
Hayden Jananthan \\ Mathematics Department \\ Vanderbilt University \\ Nashville, Tennessee \\ hayden.r.jananthan@vanderbilt.edu
}

\author{
Suna Kim \\ Mathematics Department \\ California Institute of Technology \\ Pasadena, California \\ skim3@caltech.edu
}

\author{
Jeremy Kepner \\ Lincoln Laboratory Supercomputing Center \\ Massachusetts Institute of Technology \\ Lexington, Massachusetts \\ kepner@1l.mit.edu
}

\begin{abstract}
A central problem of linear algebra is solving linear systems. Regarding linear systems as equations over general semirings $(V, \oplus, \otimes, 0,1)$ instead of rings or fields makes traditional approaches impossible. Earlier work shows that the solution space $X(\mathbf{A}, \mathbf{w})$ of the linear system $\mathbf{A v}=\mathbf{w}$ over the class of semirings called join-blank algebras is a union of closed intervals (in the product order) with a common terminal point.

In the smaller class of max-blank algebras, the additional hypothesis that the solution spaces of the $1 \times 1$ systems $A \otimes v=w$ are closed intervals implies that $X(\mathbf{A}, \mathbf{w})$ is a finite union of closed intervals. We examine the general case, proving that without this additional hypothesis, we can still make $X(\mathbf{A}, \mathbf{w})$ into a finite union of quasi-intervals.
\end{abstract}

Keywords-linear algebra, matrices, lattices, linear systems

\section{INTRODUCTION}

Linear algebra is a cornerstone of modern computation. In particular, one approach to solving a problem in application is reducing it to a linear-algebraic problem, such as carrying out a matrix multiplication, solving a linear system, or finding eigenvalues and eigenvectors.

As data become more varied, new mathematics must be developed to handle linear algebra over more general algebraic structures. For example, the need for a variety of data types to be supported exists in the context of polystore databases [1] and prompted the creation of the Dynamic Distributed Dimensional Data Model (D4M) [2] which provides a linear algebraic interface to graphs stored in NoSQL [3], [4], SQL [5], [6], and NewSQL [7].

One of the most general algebraic structures over which linear algebra makes sense is a semiring. Semirings include many algebraic structures that we often encounter - in particular, all rings and fields are semirings. Among the most studied semirings which are not rings include the max-plus algebra $\mathbb{R} \cup\{\infty,-\infty\}$, which forms a semiring with addition max and multiplication + , and the max-min algebra $\mathbb{R} \cup\{\infty,-\infty\}$, which forms a semiring with addition max and multiplication $\min [8],[9]$.

In fact, mathematicians and scientist have found numerous applications of max-plus algebra; it is widely used fields like in performance evaluation of manufacturing systems, discrete event system theory, Markov decision processes, and even in language theory [10].

Note that a semiring generalizes the notion of a ring by dropping the necessity of additive inverses existing. One method of dealing with the loss of subtraction is to make use of non-algebraic properties, particularly strong order-theoretic properties [11]. Thus we focus on the semirings that are induced from ordered sets (as in max-plus algebra) and utilize those properties to characterize the solution set.

\section{Definitions}

The most basic object of study is that of a semiring.

Definition II.1 (Semiring). [12], [13] A semiring is a quintuple $(V, \oplus, \otimes, 0,1)$ consisting of

1) an underlying set $V$,

2) two binary operations $\oplus$ (addition) and $\otimes$ (multiplication) on $V$, and

3) two elements 0 and 1 of $V$

such that

1) $\oplus$ is associative, commutative, and has identity element 0

2) $\otimes$ is associative and has identity element 1 ,

3) $\otimes$ distributes over $\oplus$, and

4) 0 is a multiplicative annihilator.

Matrices and their operations can be defined over general semirings, in a similar way it is over fields like $\mathbb{R}$ or $\mathbb{C}$.

Definition II.2 (Matrices). An $m \times n$ matrix over a semiring $V$ is a map

$$
\mathbf{A}:\{1, \ldots, m\} \times\{1, \ldots, n\} \rightarrow V
$$

If $\mathbf{A}$ and $\mathbf{B}$ are two $m \times n$ matrices, their sum is the $m \times n$ matrix $\mathbf{A} \oplus \mathbf{B}$ defined by

$$
(\mathbf{A} \oplus \mathbf{B})(i, j)=\mathbf{A}(i, j) \oplus \mathbf{B}(i, j)
$$

If $\mathbf{A}$ is an $m \times n$ matrix and $\mathbf{B}$ is an $n \times p$ matrix, their product is the $m \times p$ matrix $\mathbf{A B}$ defined by

$$
\mathbf{A B}(i, j)=\bigoplus_{k=1}^{n} \mathbf{A}(i, k) \otimes \mathbf{B}(k, j)
$$

Elements of the Cartesian product $V^{n}$ are identified with $n \times 1$ matrices over $V$.

Definition II.3 (Linear Systems). An $m \times n$ linear system over $V$ is an equation of the form $\mathbf{A v}=\mathbf{w}$ where $\mathbf{A}$ is a fixed $m \times n$ matrix, w is a fixed $m \times 1$ matrix, and $\mathbf{v}$ is a variable $n \times 1$ matrix. 
The solution space $X(\mathbf{A}, \mathbf{w})$ of a linear system $\mathbf{A v}=\mathbf{w}$ is the set

$$
X(\mathbf{A}, \mathbf{w})=\{\mathbf{v} \mid \mathbf{A} \mathbf{v}=\mathbf{w}\}
$$

One nice class of semirings which is diametrically opposite of the notion of a ring is that of join-blank algebras, which make explicit and extended use of an underlying order by requiring that the underlying set be a complete lattice, the addition operation be binary supremum, and the multiplication operation satisfy an "infinite-distributivity" law.

Definition II.4 (Complete Lattice). A pair $(V, \leq)$ of a set $V$ and a binary relation $\leq$ on $V$ is a complete lattice if

1) $\leq$ is reflexive, antistymmetric, and transitive,

2) for any subset $U \subset V$ there exists a least element $\bigvee U$ greater than or equal to every element of $U$, called the join or supremum of $U$, and

3) for any subset $U \subset V$ there exists a greatest element $\bigwedge U$ less than or equal to every element of $U$, called the meet or infimum of $U$.

In the case of a two element set $\{u, v\}$, the join of $\{u, v\}$ is denoted

$$
\bigvee\{u, v\}=u \vee v
$$

and its meet is denoted

$$
\bigwedge\{u, v\}=u \wedge v
$$

These binary operations $\vee$ and $\wedge$ are called join and meet, respectively. Semirings in which the underlying set and the operations have order-theoretic properties with respect to a fixed partial order allows for order-theoretic tools to be applied to the construction of solution sets in terms of intervals.

Definition II.5 (Join-Blank Algebra). A join-blank algebra is a semiring $(V, \vee, \otimes,-\infty, 1)$ where

1) $V$ is a complete lattice with respect to some fixed order,

2) $V$ is the join with respect to that order,

3) $-\infty$ is the minimum element of $V$ with respect to that order, and

4) for any subset $U \subset V$ and element $v \in V$

$$
v \wedge \bigvee U=\bigvee\{v \wedge u \mid u \in U\}
$$

The max-plus algebra $(\mathbb{R} \cup\{-\infty, \infty\}$, max, $+,-\infty, 0)$ and the max-min algebra $(\mathbb{R} \cup\{-\infty, \infty\}$, max, $\min ,-\infty, \infty)$ are join-blank algebras. Power set algebras $(\mathcal{P}(S), \cup, \cap, \emptyset, S)$ and more generally Heyting algebras form join-blank algebras. [14]

The order-theoretic properties of a join-blank algebra $V$ can be extended to the Cartesian product $V^{n}$.

Definition II.6 (Product Order). Suppose $V$ is ordered by $\leq$. The product order $\leq$ on $V^{n}$ is defined by

$$
\mathbf{v} \leq \mathbf{w} \quad \text { if and only if } \quad \mathbf{v}(i) \leq \mathbf{w}(i) \text { for all } i
$$

\section{Join-Blank Structure TheOREM}

The order-theoretic properties of a join-blank algebra $V$ extend to order-theoretic properties of $V^{n}$.

Proposition III.1. [14] If $V$ is a complete lattice, then $V^{n}$ is a complete lattice. Moreover, if $U \subset V^{n}$ then $\bigvee U$ exists if and only if $\bigvee\{\mathbf{v}(i) \mid \mathbf{v} \in U\}$ exists for each $i$, in which case

$$
(\bigvee U)(i)=\bigvee\{\mathbf{v}(i) \mid \mathbf{v} \in U\}
$$

The compatibility of $\vee$ and $\otimes$ with the order contribute to order-theoretic properties of $X(\mathbf{A}, \mathbf{w})$ :

Proposition III.2. [14] Suppose $\mathbf{A v}=\mathbf{w}$ is a linear system over a join-blank algebra $V$. (a)

1) $X(\mathbf{A}, \mathbf{w})$ is closed under taking joins of non-empty subsets.

2) $X(\mathbf{A}, \mathbf{w})$ is convex, so if $\mathbf{v}_{1} \leq \mathbf{v}_{2} \leq \mathbf{v}_{3}$ and $\mathbf{v}_{1}, \mathbf{v}_{3} \in$ $X(\mathbf{A}, \mathbf{w})$, then $\mathbf{v}_{2} \in X(\mathbf{A}, \mathbf{w})$.

This implies the following structure of $X(\mathbf{A}, \mathbf{w})$ as a union of closed intervals with a common terminal point. Recall that a closed interval is defined as

$$
[\mathbf{x}, \mathbf{y}]=\{\mathbf{z} \mid \mathbf{x} \leq \mathbf{z} \leq \mathbf{y}\}
$$

Theorem III.3 (Join-Blank Structure Theorem). [14] Suppose $\mathbf{A v}=\mathbf{w}$ is a linear system over a join-blank algebra $V$. Then $X(\mathbf{A}, \mathbf{w})$ is of the form

$$
X(\mathbf{A}, \mathbf{w})=\bigcup_{\mathbf{v} \in U}[\mathbf{v}, \mathbf{x}]
$$

for some $U \subset X(\mathbf{A}, \mathbf{w})$ and a fixed $\mathbf{x}$.

This structure allows for the problem of finding the solution space $X(\mathbf{A}, \mathbf{w})$ to be reduced to finding the solution spaces

$$
X(\mathbf{A}(i,:), \mathbf{w}(i))
$$

to the single-equation linear systems

$$
\mathbf{A}(i,:) \mathbf{v}=\mathbf{w}(i)
$$

Intersecting each solution set will give us the complete solution set for the original linear system, as the intersection will satisfy all equations.

\section{MaX-Blank Structure Theorem}

When $V$ is totally-ordered and hence $V=\max$, we call $V$ a max-blank algebra.

In nice cases, the solution space of a linear system over a max-blank algebra is a finite union of closed intervals. However, it is not always the case. Consider the following system in max-blank algebra:

$$
[\infty][v]=[\infty]
$$

The solution space is given by $(-\infty, \infty]$, which cannot be written as finite unions of intervals. In the $1 \times 1$ case, the solution set can be represented with finitely many closed intervals if

$$
X(A, w)=\{v \mid A \otimes v=w\}
$$


is a closed interval.

Theorem IV.1 (Max-Blank Structure Theorem for Closed Intervals). [14] Suppose $\mathbf{A v}=\mathbf{w}$ is a linear system over a max-blank algebra such that for every $i, j$ the set

$$
\{v \mid \mathbf{A}(i, j) \otimes \mathbf{w}(i)\}
$$

is a closed interval. Then $X(\mathbf{A}, \mathbf{w})$ is a finite union of closed intervals.

The crucial step in the proof of Theorem IV.1 is that a Cartesian product of closed intervals in $V$ is a closed interval in $V^{n}$ in the product order.

Proposition III.2 shows that the only other form that

$$
\{v \mid \mathbf{A}(i, j) \otimes \mathbf{w}(i)\}
$$

can take on is a half-open interval which is open on the left. The Cartesian product of arbitrary intervals in $V$ need to be an interval in $V^{n}$ in the product order.

This motivates a slightly more general basic object than intervals.

Definition IV.2 (Quasi-interval). Suppose $I_{1}, \ldots, I_{n}$ are intervals in $V$. Then define

$$
{ }_{A}[\mathbf{p}, \mathbf{q}]_{B}=I_{1} \times \cdots \times I_{n}
$$

where $I_{k}$ has endpoints $\mathbf{p}(k)$ and $\mathbf{q}(k)$, with exclusion of $\mathbf{p}(k)$ when $k \in A$ and exclusion of $\mathbf{q}(k)$ when $k \in B$.

Lemma IV.3. Suppose $V$ is totally ordered. Suppose $A, B \subset$ $\{1, \ldots, n\}$ and $\mathbf{p}, \mathbf{q}, \mathbf{r}, \mathbf{s} \in V^{n}$. Then

$$
{ }_{A}[\mathbf{p}, \mathbf{q}] \cap{ }_{B}[\mathbf{r}, \mathbf{s}]={ }_{C}[\mathbf{p} \vee \mathbf{r}, \mathbf{q} \wedge \mathbf{s}]
$$

where

$$
\begin{aligned}
C= & \{i \in A \backslash B \mid \mathbf{p}(i) \geq \mathbf{r}(i)\} \\
& \cup\{j \in B \backslash A \mid \mathbf{p}(j) \leq \mathbf{r}(j)\} \\
& \cup A \cap B
\end{aligned}
$$

Proof: Let

$$
{ }_{A}[\mathbf{p}, \mathbf{q}]=I_{1} \times \cdots \times I_{n}
$$

and

$$
{ }_{B}[\mathbf{r}, \mathbf{s}]=J_{1} \times \cdots \times J_{n}
$$

Then

$$
\begin{aligned}
{ }_{A}[\mathbf{p}, \mathbf{q}] \cap{ }_{B}[\mathbf{r}, \mathbf{s}] & =\left(I_{1} \times \cdots \times I_{n}\right) \cap\left(J_{1} \times \cdots \times J_{n}\right) \\
& =\left(I_{1} \cap J_{1}\right) \times \cdots \times\left(I_{n} \cap J_{n}\right)
\end{aligned}
$$

Since the intersection of intervals is also interval, each of $I_{k} \cap$ $J_{k}$ is an interval with end-points $\mathbf{p}(k) \vee \mathbf{r}(k)$ and $\mathbf{q}(k) \wedge \mathbf{s}(k)$ with exclusion of the first end-point $\mathbf{p}(k) \vee \mathbf{r}(k)$ exactly when either (i)

1) $k \in A \backslash B$ and $\mathbf{p}(k) \geq \mathbf{r}(k)$, or

2) $k \in B \backslash A$ and $\mathbf{p}(k) \leq \mathbf{r}(k)$, or

3) $k \in A \cap B$. and inclusion of the second end-point $\mathbf{q}(k) \wedge \mathbf{s}(k)$. Hence

$$
{ }_{A}[\mathbf{p}, \mathbf{q}] \cap{ }_{B}[\mathbf{r}, \mathbf{s}]={ }_{C}[\mathbf{p}, \mathbf{q}]
$$

where

$$
\begin{aligned}
C= & \{i \in A \backslash B \mid \mathbf{p}(i) \geq \mathbf{r}(i)\} \\
& \cup\{j \in B \backslash A \mid \mathbf{p}(j) \leq \mathbf{r}(j)\} \\
& \cup(A \cap B)
\end{aligned}
$$

Using this new notation, we show that linear systems in max-blank algebra have solution set that can be written as union of finite quasi-interval.

Theorem IV.4 (Max-blank Structure Theorem). Suppose A is an $n \times m$ matrix and $\mathbf{w}$ an element of $V^{n}$. Let $U_{i}, U_{i, 1}$, and $U_{i, 2}$ be the sets of $j \in\{1, \ldots, m\}$ such that

$$
X(\mathbf{A}(i, j), \mathbf{w}(i))=\{v \in V \mid \mathbf{A}(i, j) \otimes v=\mathbf{w}(i)\}
$$

is non-empty, non-empty and not a closed interval, and non-empty and a closed interval, respectively. When $j \in$ $U_{i, 1}$, let $X(\mathbf{A}(i, j), \mathbf{w}(i))=\left(p_{j}^{i}, q_{j}^{i}\right]$. For $j \in U_{i, 2}$, let $X(\mathbf{A}(i, j), \mathbf{w}(i))=\left[p_{j}^{i}, q_{j}^{i}\right]$. Lastly, for $j \notin U_{i}$ let $q_{j}^{i}$ be the largest element such that $\mathbf{A}(i, j) \otimes q_{j}^{i} \leq \mathbf{w}(i)$.

Let $\mathbf{p}_{i, j}$ be defined by

$$
\mathbf{p}_{i, j^{\prime}}(j)= \begin{cases}p_{j^{\prime}}^{i} & j=j^{\prime} \\ -\infty & \text { otherwise }\end{cases}
$$

and $\mathbf{q}_{i}$ be defined by $\mathbf{q}_{i}(j)=q_{j}^{i}$. Then

$$
\left.X(\mathbf{A}, \mathbf{w})=\bigcup_{\substack{j_{i, k}^{\prime} \in U_{i, k} \\ \text { for } 1 \leq i \leq n \\ \text { and } k \in\{1,2\}}} \bigvee_{J_{\mathbf{j}} \in i \leq n, k \in\{1,2\}} \mathbf{p}_{i, j_{i, k}^{\prime}}, \bigwedge_{1 \leq i \leq n} \mathbf{q}_{i}\right]
$$

where $\mathbf{j}=\left(j_{i, k}^{\prime}\right)_{1 \leq i \leq n, k \in\{1,2\}}$ and $\ell \in J_{\mathbf{j}}$ if and only if

$$
\max _{1 \leq i \leq n} \mathbf{p}_{i, j_{i, 2}} \leq \max _{1 \leq i \leq n} \mathbf{p}_{i, j_{i, 1}}
$$

Proof: The proof will consist of finding the solution space of $\max _{j \in\{1, \ldots, m\}}(\mathbf{A}(i, j) \otimes \mathbf{v}(j))=\mathbf{w}(i)$ for each $i \in\{1, \ldots, n\}$ and showing that it is the union of intervals with a common (inclusive) terminal point. Taking the intersection of these solution spaces is $X(\mathbf{A}, \mathbf{w})$.

$X([v],[u])$ is convex with a inclusive terminal point. Since $V$ is a complete lattice, it follows that

$\left.X(\mathbf{A}(i, j), \mathbf{w}(i))=\left[p_{j}^{i}, q_{j}^{i}\right]\right) \quad$ or $\quad X(\mathbf{A}(i, j), \mathbf{w}(i))=\left(p_{j}^{i}, q_{j}^{i}\right]$ for some $p_{j}^{i}$ and $q_{j}^{i}$, assuming that $X(\mathbf{A}(i, j), \mathbf{w}(i))$ is nonempty.

Let define $U_{i}, U_{i, 1}$, and $U_{i, 2}$ as in the proposition. For $j \notin U_{i}$, let $q_{j}^{i}$ be the largest element such that $\mathbf{A}(i, j) \otimes q_{j}^{i} \leq$ $\mathbf{w}(i)$. Such an element exists since $-\infty \otimes v=-\infty$ and multiplication by a fixed element is a monotonic map.

The solution space now can be written down in terms of the elements $p_{j}^{i}$ and $q_{j}^{i}$. A given $\mathbf{v}$ is in the solution set if and only if there exists a $j^{\prime} \in\{1, \ldots, m\}$ such that

$$
\mathbf{A}\left(i, j^{\prime}\right) \otimes \mathbf{v}\left(j^{\prime}\right)=\mathbf{w}(i)
$$


and for all $j \in\{1, \ldots, m\}$ it is true that

$$
\mathbf{A}(i, j) \otimes \mathbf{v}(j) \leq \mathbf{w}(i)
$$

The first condition is that $\mathbf{v}\left(j^{\prime}\right) \in f_{\mathbf{A}\left(i, j^{\prime}\right)}^{-1}(\mathbf{w}(i))$ and the second condition is that $\mathbf{v}(j) \in\left[-\infty, q_{j}^{i}\right]$ because multiplication by a fixed element is a monotonic function. Then the solution space can be written as

$$
\begin{array}{r}
\bigcup_{j^{\prime} \in U_{i}}\left(X\left(\mathbf{A}\left(i, j^{\prime}\right), \mathbf{w}(i)\right) \times \prod_{j \in\{1, \ldots, m\}, j \neq j^{\prime}}\left[-\infty, q_{j}^{i}\right]\right) \\
=\bigcup_{j^{\prime} \in U_{i, 1}}\left(\left(p_{j^{\prime}}^{i}, q_{j^{\prime}}^{i}\right] \times \prod_{j \in\{1, \ldots, m\}, j \neq j^{\prime}}\left[-\infty, q_{j}^{i}\right]\right) \\
\cup \bigcup_{j^{\prime} \in U_{i, 2}}\left(\left[p_{j^{\prime}}^{i}, q_{j^{\prime}}^{i}\right] \times \prod_{j \in\{1, \ldots, m\}, j \neq j^{\prime}}\left[-\infty, q_{j}^{i}\right]\right)
\end{array}
$$

Taking $\mathbf{p}_{i, j^{\prime}}(j)$ and $\mathbf{q}_{i}(j)$ as defined before, we get

$$
\left[p_{j^{\prime}}^{i}, q_{j^{\prime}}^{i}\right] \times \prod_{j \in\{1, \ldots, m\}, j \neq j^{\prime}}\left[-\infty, q_{j}^{i}\right]=\left[\mathbf{p}_{i, j^{\prime}}, \mathbf{q}_{i}\right]
$$

and

$$
\left(p_{j^{\prime}}^{i}, q_{j^{\prime}}^{i}\right] \times \prod_{j \in\{1, \ldots, m\}, j \neq j^{\prime}}\left[-\infty, q_{j}^{i}\right]={ }_{j^{\prime}}\left[\mathbf{p}_{i, j^{\prime}}, \mathbf{q}_{i}\right]
$$

Let

$$
I_{i, j}= \begin{cases}\{j\} & \text { if } j \in U_{i, 1} \\ \emptyset & \text { otherwise }\end{cases}
$$

Using this, we can change the order of intersection with union to get

$$
\begin{aligned}
& X(\mathbf{A}, \mathbf{w})=\bigcap_{i=1}^{n}\left(\bigcup_{j^{\prime} \in U_{i, 1}} j^{\prime}\left[\mathbf{p}_{i, j^{\prime}}, \mathbf{q}_{i}\right] \cup \bigcup_{j^{\prime} \in U_{i, 2}}\left[\mathbf{p}_{i, j^{\prime}}, \mathbf{q}_{i}\right]\right) \\
& \left.=\bigcup_{j_{i, k}^{\prime} \in U_{i, k}} \bigcap_{1 \leq i \leq n, k \in\{1,2\}} I_{i, j_{i, k}^{\prime}} \llbracket \mathbf{p}_{i, j_{i, k}^{\prime}}, \mathbf{q}_{i}\right] \\
& \text { for } 1 \leq i \leq n \\
& \text { and } k \in\{1,2\}
\end{aligned}
$$

Finally, since the intersection of quasi-intervals can be represented as a quasi-interval as well (LemmaIV.3), we arrive at

$$
\left.X(\mathbf{A}, \mathbf{w})=\bigcup_{\substack{j_{i, k}^{\prime} \in U_{i, k} J_{\mathbf{j}} \\ \text { for } 1 \leq i \leq n \\ \text { and } k \in\{1,2\}}} \bigvee_{1 \leq i \leq n, k \in\{1,2\}} \mathbf{p}_{i, j_{i, k}^{\prime}}, \bigwedge_{1 \leq i \leq n} \mathbf{q}_{i}\right]
$$

where $\mathbf{j}=\left(j_{i, k}^{\prime}\right)_{1 \leq i \leq n, k \in\{1,2\}}$ and $\ell \in J_{\mathbf{j}} \subset\{1, \ldots, n\}$ if and only if

$$
\max _{1 \leq i \leq n} \mathbf{p}_{i, j_{i, 2}^{\prime}}(\ell) \leq \max _{1 \leq i \leq n} \mathbf{p}_{i, j_{i, 1}^{\prime}}(\ell)
$$

\section{FURTHER RESEARCH}

The notion of a quasi-interval allows the structure of the solution space of a linear system over a max-blank algebra to be written as a finite union of quasi-intervals. This naturally leads to the question of how this notion can be used to express other solution spaces in similarly nice ways.

While the maximum solution of a linear system is known in many cases, particularly for Heyting algebras (a join-blank algebra in which $\otimes$ is the meet) and max-blank algebras, the entire structure is not known for arbitrary Heyting algebras.

Also worth investigating is how crucial each of the properties a join-blank algebra satisfies are to Theorem III.3.

\section{REFERENCES}

[1] J. Kepner, V. Gadepally, D. Hutchison, H. Jananthan, T. Mattson, S. Samsi, \& A. Reuther. "Associative Array Model of SQL, NoSQL, and NewSQL Databases." CoRR 1606.05797, 2016

[2] J. Kepner, W. Arcand, W. Bergeron, N. Bliss, R. Bond, C. Byun, G. Condon, K. Gregson, M. Hubbell, J. Kurz, A. McCabe, P. Michaleas, A. Prout, A. Reuther, A. Rosa \& C. Yee, "Dynamic Distributed Dimensional Data Model (D4M) Database and Computation System," ICASSP (International Conference on Accoustics, Speech, and Signal Processing), 2012, Kyoto, Japan

[3] C. Byun, W. Arcand, D. Bestor, B. Bergeron, M. Hubbell, J. Kepner, A. McCabe, P. Michaleas, J. Mullen, D. OGwynn, A. Prout, A. Reuther, A. Rosa, \& C. Yee, "Driving Big Data With Big Compute," IEEE High Performance Extreme Computing (HPEC) Conference, Sep 2012

[4] J. Kepner, C. Anderson, W. Arcand, D. Bestor, B. Bergeron, C. Byun, M. Hubbell, P. Michaleas, J. Mullen, D. O'Gwynn, A. Prout, A. Reuther, A. Rosa, \& C. Yee, "D4M 2.0 Schema: A General Purpose High Performance Schema for the Accumulo Database," IEEE High Performance Extreme Computing (HPEC) conference, Sep 10-12, 2013, Waltham, MA

[5] S. Wu, V. Gadepally, A. Whitaker, J. Kepner, B. Howe, M. Balazinska \& S. Madden, "MIMICViz: Enabling Visualization of Medical Big Data," Intel Science \& Technology Center retreat, Portland, OR, August, 2014

[6] V. Gadepally, J. Kepner, W. Arcand, D. Bestor, B. Bergeron, C. Byun, L. Edwards, M. Hubbell, P. Michaleas, J. Mullen, A. Prout, A. Rosa, C. Yee, \& A. Reuther, "D4M: Bringing Associative Arrays to Database Engines", IEEE High Performance Extreme Computing (HPEC) conference, Sep 2015, Waltham, MA

[7] S. Samsi, L. Brattain, V. Gadepally, \& J. Kepner "D4M and Large Array Databases for Management and Analysis of Large Biomedical Imaging Data," New England Database Summit, 2016

[8] M. Akian, R. Bapat, \& S. Gaubert. "Max-plus algebra." Handbook of linear algebra (discrete mathematics and its applications) 39 (2006): 1014.

[9] G. L. Litvinov \& S. N. Sergeev, eds. Tropical and Idempotent Mathematics: International Workshop Tropical-07, Tropical and Idempotent Mathematics, August 25-30, 2007, Independent University of Moscow and Laboratory J.-V. Poncelet. Vol. 495. American Mathematical Soc., 2009.

[10] S. Gaubert. "Methods and applications of $(\max ,+)$ linear algebra." Annual Symposium on Theoretical Aspects of Computer Science. Springer, Berlin, Heidelberg, 1997

[11] J. S. Han, H. S. Kim, \& J. Neggers. "Semiring orders in a semiring." Appl. Math. \& Inform. Sci 6 (2012): 99-102.

[12] J. Golan. "Semirings and their Applications", Kluwer Academic Publishers, 1999

[13] M. Gondran \& M. Minoux. "Dioids and semirings: Links to fuzzy sets and other applications." Fuzzy Sets and Systems 158.12 (2007): 12731294.

[14] J. Kepner \& H. Jananthan. "Mathematics of Big Data", MIT Press, 2018 